Running title: Navigational efficiency in a BCRW model

\title{
Navigational efficiency in a biased and correlated random walk model of individual animal
} movement

Joseph D. Bailey ${ }^{1}$, Jamie Wallis ${ }^{1,2}$, Edward A. Codling ${ }^{1 \dagger}$

${ }^{1}$ Department of Mathematical Sciences, University of Essex, Colchester CO4 3SQ, UK. ${ }^{2}$ Institute of Biomedical Engineering, University of Oxford, Oxford, OX3 7DQ, UK.

Abstract: Understanding how an individual animal is able to navigate through its environment is a key question in movement ecology that can give insight into observed movement patterns and the mechanisms behind them. Efficiency of navigation is important for behavioural processes at a range of different spatio-temporal scales, including foraging and migration. Random walk models provide a standard framework for modelling individual animal movement and navigation. Here we consider a vector-weighted biased and correlated random walk (BCRW) model for directed movement (taxis), where external navigation cues are balanced with forward persistence. We derive a mathematical approximation of the expected navigational efficiency for any BCRW of this form and confirm the model predictions using simulations. We demonstrate how the navigational efficiency is related to the weighting given to forward persistence and external navigation cues, and highlight the counter-intuitive result that for low (but realistic) levels of error on forward persistence, a higher navigational efficiency is achieved by giving more weighting to this indirect navigation cue rather than direct navigational cues. We discuss and interpret the relevance of these results for understanding animal movement and navigation strategies.

Keywords: Animal movement; biased and correlated random walk (BCRW); movement ecology; navigation; persistence.

${ }^{\dagger}$ Contact author: ecodling@essex.ac.uk 


\section{Introduction}

Understanding how and why animals navigate through their environment is one of the key open questions in movement ecology (Nathan, 2008). Animal navigation can occur across various spatio-temporal scales corresponding to a wide variety of behavioural processes, ranging from short-term foraging and home-range exploration (Schultz \& Crone, 2001; Fortin et al., 2005; McClintock et al., 2012) to large-scale migration events (Gardiner et al., 2015). Common navigation orientation mechanisms include taxis, where an animal directly orientates in response to external directional cues, and differential klinokinesis (DKK), where the level of turning in the movement path (sinuosity) depends on variations in the magnitude of an external stimulus; both taxis and DKK lead to a long-term directional drift (bias) towards the target (Benhamou \& Bovet, 1992). Conversely, the short-term localised bias in movement directions due to the tendency of animals to continue moving in the same direction is known as forward persistence (Benhamou \& Bovet, 1992). Empirical studies have considered how a variety of different animals may balance taxis and persistence mechanisms in order to navigate within their local environment, including butterflies (Schultz \& Crone, 2001), elk (Fortin et al., 2005), and grey seals (McClintock et al., 2012). Hence it is important to consider a theoretical basis for animal navigation and the underlying mechanisms that may lead to improved navigational efficiency.

Random walk theory has a long history of being used to model individual animal movement and navigation, and as a tool to classify and interpret observed movement data using various path analysis techniques (Jonsen et al., 2005; Codling et al., 2008; Langrock et al., 2012; McClintock et al., 2012). Models of movement based purely on localised forward persistence are known as correlated random walks (CRW) (Kareiva and Shigesada, 1983; Bovet \& Benhamou, 1988; Codling et al., 2008). Biased random walks (BRW) are movement models where there is a 
long term directional bias in the direction of movement (Marsh and Jones, 1988; Benhamou, 2006; Codling et al., 2008; Codling et al., 2010). Models that combine both long-term directional bias and forward persistence are known as biased and correlated random walks (BCRW) (Codling et al., 2008).

Benhamou \& Bovet (1992) combined taxis and forward persistence to form a vectorweighted BCRW model of movement, and found in a 1000-step random walk that the best navigational strategy was to give approximately $10 \%$ weighting to external navigation cues (taxis) and approximately $90 \%$ weighting to forward persistence. Such a navigation strategy gave higher navigational efficiency than a movement process based on taxis alone (a pure BRW), although it should be stressed that Benhamou \& Bovet (1992) only included error in their external navigation term and not in their forward persistence term. The fact that the most efficient navigation strategy involved giving a high weighting to persistence rather than taxis may seem to be a counter-intuitive result, since movement based purely on persistence is known to be an inefficient navigation strategy when compared to pure taxis (Cheung et al., 2007). A similar result to Bovet \& Benhamou (1992) was obtained by Codling \& Bode (2014) who found that, in the context of a collective movement model for navigating animal groups, the most efficient navigational strategy was to give a high weighting to indirect navigational cues (copying the movement of other group members) and a low (but non-zero) weighting to direct individual navigational cues (taxis). In a follow-up study, Codling \& Bode (2016) included individual forward persistence in the collective movement model and showed that giving a high weighting to indirect cues (copying neighbours or using forward persistence) rather than relying on direct navigational cues gave the highest navigational efficiency. 
The findings of Benhamou \& Bovet (1992) and Codling \& Bode $(2014,2016)$ were based on simulations only and the authors did not give a mathematical explanation for these results. Here we consider a generalised form of the Benhamou \& Bovet (1992) BCRW navigation model, that includes error on the persistence term, and derive a mathematical approximation for the expected navigational efficiency. The model predicts that for a reasonably large (and realistic) range of navigation and persistence errors, the highest navigational efficiency is achieved by giving a low weighting to direct navigational cues. We discuss the relevance and implications of these findings in the wider movement ecology context.

\section{Mathematical model}

The BCRW model of Benhamou \& Bovet (1992) assumes a single random walker starts at the origin, $(0,0)$, and moves through an empty homogenous two-dimensional environment. For simplicity, the target is assumed to be a 'point at infinity' located along the positive $x$-axis (this effectively means we are only considering the large-scale part of the navigation process when the animal is far from the target). Orientation angles are measured counter-clockwise from the $x$ axis, and hence the target direction is given by $\Omega_{T}=0$. We assume the walker initially starts with no information about the target direction; an initial movement direction, $\theta_{0}$, is randomly drawn from a uniform circular distribution (this assumption does not affect our results as we will show that the long-term navigational efficiency is independent of $\theta_{0}$ ). At each random walk step the components of movement in each direction are given by a weighted vector sum of a navigation term and a persistence term (Benhamou \& Bovet, 1992):

$$
\begin{aligned}
\Delta x_{n+1} & =r_{n+1}\left(w \cos \left(\Omega_{T}+\phi_{n}\right)+(1-w) \cos \left(\theta_{n}+\delta_{n}\right)\right) \\
\Delta y_{n+1} & =r_{n+1}\left(w \sin \left(\Omega_{T}+\phi_{n}\right)+(1-w) \sin \left(\theta_{n}+\delta_{n}\right)\right)
\end{aligned}
$$


where $r_{n+1}$ is the step length (distance moved) in the current step, $\Omega_{T}=0$ is the target direction (which is fixed as the $x$-axis for all steps), $\theta_{n}$ is the direction of movement in the previous step (which varies at each step), $\delta_{n}$ is a persistence error term, $\phi_{n}$ is a navigation error term, and $w \in[0,1]$ is the weighting given to navigation (and hence $(1-w)$ is the weighting given to persistence). In contrast to Benhamou \& Bovet (1992) (who only included an error in the navigation term), we include errors on both the navigation and persistence terms. In principle it would be possible to have an even more general BCRW model that includes an additional 'output noise/error' term in addition to the navigation and persistence error terms. This output noise could represent either additional movement error because of environmental factors (e.g. turbulence) or could represent a form of 'voluntary' error that the animal may use to modulate its behaviour between behavioural states. However, these more complex model features are beyond the scope of the current work.

The additive navigation and persistence random error terms, $\phi_{n}$ and $\delta_{n}$, are drawn from separate zero-centred symmetric circular distributions with respective mean cosine values given by $c_{\phi} \in[0,1]$ and $c_{\delta} \in[0,1]$. The level of navigation and/or persistence error is determined by the mean cosine values, $c_{\phi}$ and $c_{\delta}$ : a value close to 0 corresponds to very high error, and a value close to 1 corresponds to very low error. We assume that $c_{\phi}$ and $c_{\delta}$ are fixed for all steps of the random walk, which implies that direction and persistence errors are independent (no correlation of errors between successive steps) and are not related to spatial location or any other external factor. Hence we do not consider possible changes in navigation cue strength as the animal approaches the target, interactions with other animals, or changes in behaviour and interactions with the environment such as foraging or resting during the navigation process. Note that, as long as $c_{\phi}$ and $c_{\delta}$ are defined, the choice of which circular distribution to use is not important 
since the results only depend on the first trigonometric moment (the mean cosine value); the same results are obtained using common circular distributions such as the wrapped normal, von Mises and wrapped Cauchy (Mardia \& Jupp, 1999).

Similar to Benhamou \& Bovet (1992), we define the navigational efficiency of a single step of the movement process as:

$$
\text { Navigational efficiency }=\frac{\text { Net distance moved towards target in } x \text { direction }}{\text { Total distance moved }} .
$$

Hence, for the BCRW given by Eqs. (1) and (2) the expected navigational efficiency at a given step is given by $\mathrm{E}\left[\cos \theta_{\mathrm{n}+1}\right]$ since the target direction is the $x$-axis $\left(\Omega_{T}=0\right)$. In the extreme case of a pure BRW $(w=1)$, navigational efficiency is given exactly by $\mathrm{E}\left[\cos \phi_{n}\right]=c_{\phi}$, while for a pure CRW $(w=0)$, navigational efficiency is given exactly by $\mathrm{E}\left[\cos \left(\theta_{n}+\delta_{n}\right)\right]=0$, since we assume a uniform initial orientation and there is no external navigation cue. More generally for 0 $<w<1$, an expression for $\mathrm{E}\left[\cos \theta_{\mathrm{n}+1}\right]$ is found by normalising Eq. (1) relative to the total step length (noting that $r_{n+1}$ cancels and that $\Omega_{T}=0$ ) and then taking the expectation:

$$
E\left[\cos \theta_{n+1}\right]=E\left[\frac{w \cos \phi_{n}+(1-w) \cos \left(\theta_{n}+\delta_{n}\right)}{\sqrt{w^{2}+(1-w)^{2}+2 w(1-w) \cos \left(\phi_{n}-\left(\theta_{n}+\delta_{n}\right)\right)}}\right]
$$

The expression on the right-hand side of Eq. (4) is non-linear so we cannot directly calculate the expectation. To make progress we use a similar argument to Wu et al. (2000), and assume we can treat the right-hand side of Eq. (4) as if it were linear. We then further assume that $\theta_{n}, \phi_{n}$ and $\delta_{n}$ are all independent, and based on a result from Hill \& Häder (1997), we assume that in the long-term limit, the distribution of movement directions is stable such that $E\left[\cos \theta_{n+1}\right]=$ $E\left[\cos \theta_{n}\right]=E\left[\cos \theta_{\infty}\right] \equiv c_{\theta}$, which is equivalent to the long-term navigational efficiency. This leads to a cubic polynomial for $c_{\theta}$ (see Appendix S1 for full details of the derivation): 


$$
2 w(1-w) c_{\phi} c_{\delta} c_{\theta}^{3}+\left(w^{2}+(1-w)^{2}\left(1-c_{\delta}^{2}\right)\right) c_{\theta}^{2}-2 w(1-w) c_{\phi} c_{\delta} c_{\theta}-w^{2} c_{\phi}^{2}=0 .
$$

It is possible to show that this polynomial has precisely one root in $[0,1]$ for all feasible values of $c_{\phi}, c_{\delta}$ and $w$ (see Appendix S2). This root can then be calculated using Cardano's method:

$$
c_{\theta}=\sqrt[3]{A+\sqrt{A^{2}+B^{3}}}+\sqrt[3]{A-\sqrt[2]{A^{2}+B^{3}}}-C
$$

where $\mathrm{A}, \mathrm{B}$, and $\mathrm{C}$ are terms involving $w, c_{\phi}$, and $c_{\delta}$, and are given in Appendix S1.

\section{Results}

We first consider the specific case of zero error on forward persistence, $c_{\delta}=1$, which was considered originally by Benhamou \& Bovet (1992). In this case Eq. (5) can be simplified and a limiting argument can be used to show that for all $c_{\phi}>0$ (i.e. as long as external navigation cues are present) then $c_{\theta} \rightarrow 1$ as $w \rightarrow 0$ (see Appendix S3 and Figure 1a-c). It may seem counter-intuitive that more efficient navigation can be obtained by giving a vanishingly small (but strictly non-zero) weighting to direct navigational cues but this is explained by the fact that once a navigating animal is oriented towards the target direction (which is always eventually possible if $w$ is strictly non-zero), it can then maintain this direction of movement indefinitely since there is no persistence error, and hence no further external navigation cues are required. Nevertheless, the higher the level of navigation error (i.e. the lower the value of $c_{\phi}$ ) the longer it will take (on average) for the animal to orientate itself towards the target direction. Consequently, the apparent navigational efficiency is highly dependent on the number of steps in the observed movement process when there is zero persistence error (Figure 1a-c). Benhamou \& Bovet (1992) only considered simulation results for a 1000-step BCRW and hence didn't report this long-term limit result directly. For $w>0$, the predicted long-term navigational efficiency, 
$c_{\theta}$, monotonically decreases as $w$ increases, indicating that lower long-term navigational efficiency is obtained when giving a higher weight to external navigation cues. This result is confirmed with the simulation results for the 10,000 step BCRW shown in Figure 1c. In Figure 1a and $1 \mathrm{~b}$ where the BCRW runs for only 100 or 1000 steps respectively, the curves showing the simulated navigational efficiency are non-monotonic: the navigational efficiency is dependent on $c_{\phi}$ with a peak for $0<w<0.2$ similar to the result reported by Benhamou \& Bovet (1992). This indicates that even with zero error on forward persistence, a BCRW with a small number of steps requires external navigational cues for efficient navigation. $\% \%$ Figure 1 about here $\% \%$

In the case where there is error on both the navigation and persistence components of the movement process (i.e. $c_{\delta}, c_{\phi}<1$ ), the solution given in Eq. (6) predicts that as the persistence error increases $\left(c_{\delta}\right.$ decreases), the navigational efficiency also decreases for all $c_{\phi}$ (solid lines in Figure 1c-f). The predicted solution curves are (in general) non-monotonic with a peak indicating a maximum navigational efficiency for an intermediate value of $w$. For low persistence errors $\left(c_{\delta}=0.99,0.95,0.9\right.$ in Figure 1d-f) the maximum navigational efficiency occurs for $w<0.5$, and at lower values of $w$ when $c_{\phi}$ is also small (higher navigation error). As the persistence error increases $\left(c_{\delta}=0.7,0.5,0.1\right.$ in Figure $1 \mathrm{~g}$-i) the peak indicating the maximum navigational efficiency shifts to the right corresponding to a larger value of the weighting given to navigation, $w$ (Figure 1g-i; Appendix S4). In general, the simulation results shown in Figure 1g-i show the same qualitative behaviour as the predicted solution curves from Eq. (6). In contrast to the case of zero persistence error, for the values of $c_{\delta}<1$ considered here, there is very little difference in the model predictions and simulated results for $n=100,1000$ and 10,000 steps (see Appendix S5: Figure S1). The inclusion of even a small error on the persistence term means the effective 
long-term limiting solutions for navigational efficiency are reached much faster than the case with zero persistence error.

\section{$\% \%$ Figure 2 about here $\% \%$}

The location of the peak corresponding to the maximum navigational efficiency for each of the curves in Figure 1d-i can be calculated directly from Eqs. (5) and (6). More generally, it can be shown that Eq. (6) has precisely one turning point for $w \in[0,1]$, and this turning point corresponds to the maximum predicted navigational efficiency (see Appendix S4). The values of $w$ leading to the theoretical maximum navigational efficiency, together with the predicted values for this efficiency, are calculated from Eqs. (5) and (6) for a range of $c_{\phi}$ and $c_{\delta}$, and are shown in Figure $2 \mathrm{a}$ and $2 \mathrm{~b}$ respectively. Figure $2 \mathrm{c}$ and $2 \mathrm{~d}$ show the equivalent results from simulations of the BCRW, and show a good qualitative match to the theoretical predictions. Figure $2 \mathrm{a}$ and $2 \mathrm{c}$ highlight the result (also seen in Figures 1d-i) that when the persistence error is high $\left(c_{\delta}<0.1\right)$, the maximum navigational efficiency occurs when giving a higher weighting to the navigation term ( $w>0.9$ ), and when $c_{\delta} \approx 0$ this efficiency is given exactly by $c_{\phi}$ (Figure $2 \mathrm{~b}$ and $2 \mathrm{~d}$ ) which corresponds to pure taxis. However, when the persistence error is low $\left(c_{\delta}>0.9\right)$, the maximum navigational efficiency typically occurs for $w<0.5$ (more weighting on persistence than direct navigation) even if the navigation error is also low (Figures $2 a$ and $2 c$ ). Figure $2 b$ and $2 d$ highlight that a high navigational efficiency can be maintained even when the navigation error is large $\left(c_{\phi}<0.5\right)$ because of the weighting given to persistence: the contour corresponding to a long-term navigational efficiency of 0.5 extends well below $c_{\phi}<0.5$ (Figures $2 \mathrm{~b}$ and $2 \mathrm{~d}$ ).

\section{Discussion}



and derived a mathematical approximation for the long-term navigational efficiency of this form of BCRW. We have demonstrated how navigational efficiency depends on the weighting given between navigation and persistence at each step of the movement process and the level of error in each term (Eq. 6; Figure 1d-i). In Figure 2a, the area above the contour line corresponding to $w=0.5$ indicates the region of the parameter space where giving a lower weighting to direct navigation cues and a higher weighting to persistence leads to the maximum long-term navigational efficiency. Interestingly, the predicted contour line corresponding to $w=0.5$ approaches $c_{\phi}=1$ in Figure 2a, indicating that even with close to zero error on the navigation cue, there is little loss of navigational efficiency by giving equal weight to persistence. This potentially hints at some interesting evolutionary advantages for animals that balance forward persistence and external navigation cues in this way: giving a high weighting to persistence can improve overall navigation efficiency if the error on navigation cues is high $\left(c_{\phi}<0.5\right)$, and has little detrimental effect if the error on navigation cues is low $\left(c_{\phi}>0.9\right)$. The vector-weighted BCRW navigation model discussed by Benhamou \& Bovet (1992) did not include error on the persistence term and the results given in their paper were for a BCRW with only 1000 steps. We have shown mathematically (Appendix S3 and Figure 1c) that in this scenario the long-term maximum navigation efficiency is actually obtained when the weighting on external navigation cues (taxis) tends to zero, i.e. $c_{\theta} \rightarrow 1$ as $w \rightarrow 0$. This may seem like a counter-intuitive result, given that a movement process based purely on persistence with no taxis is known to be a poor navigation strategy (Cheung, 2007). However, this result holds only for the long-term limiting navigation efficiency, where the fact that there is zero error on persistence means an animal can achieve maximum navigational efficiency simply by continuing 
in the same direction as previously once it is moving in the target direction. At shorter timescales navigation will not be as efficient (Figures 1a-c), and the observed navigational efficiency is dependent on the number of steps of the BCRW. A possible further extension of this work would be to derive an expression for the navigational efficiency that is valid for a small number of steps and not just the long-term limit. However, Appendix S5: Figure S1 demonstrates how the sensitivity of the navigational efficiency to the number of steps in the BCRW is less when persistence error is non-zero. When referring to the results in Figures 1a-c we are implicitly assuming that a random walk with 1000 steps is 10 times longer than a walk with 100 steps and so on. Instead, an alternative interpretation of the model and results is that the overall path length is fixed and it is the reorientation frequency that changes (so that a single step in a 1000-step path is 10 times shorter than that in a 100-step path). From Figure 1a-c, this then implies that in a noisy environment an animal may improve its navigational efficiency by increasing its rate of reorientation (effectively giving a larger sample size in the navigational averaging process).

The BCRW model considered here is deliberately simple but could easily be extended to consider more complex scenarios, although this may come at the expense of analytical tractability, and generalised predictions about navigation efficiency informed by mathematical theory may not subsequently be possible. For example, our model assumes individuals do not interact with conspecifics but the overall conclusions are similar to results observed by Codling \& Bode $(2014,2016)$ who used simulations to demonstrate that in social group navigation, the most efficient navigational strategy was to give a high weighting to indirect cues (copying the movement of other group members or using forward persistence), rather than relying on direct navigational cues (taxis). Our BCRW model assumes a homogeneous environment with a fixed target direction where navigation cues and errors do not vary in space or time. Additionally, 
individual walkers do not change their movement behaviour over time or when in different spatial locations. Many migrating animals will undertake other behaviours during the large-scale navigation process (such as resting, foraging etc), or may change their behaviour in response to their local environment. Peleg and Mahadevan (2015) developed a random walk model which includes periods of purely persistent behaviour followed by a pause to reorient according to external navigational cues. By repeating this behaviour, a walk which appears to be purely persistent (a CRW) in the short term can then be described as a BCRW in the long term. This is in contrast to our model where we assume the animal is continuously balancing persistence and navigation (taxis) at every step of the movement path. Nevertheless, the relative weighting between navigation and persistence in our model $(w)$ can be directly compared to the relative navigation reorientation frequency in the model of Peleg and Mahadevan (2015) since both effectively give a way to balance persistence and navigation. In the context of group navigation, Bode et al. (2010) used a similar approach and considered a model where the behaviour of an individual at each step was probabilistic and chosen to be either purely persistent or purely local navigation. The probability of choosing persistence against navigation in this model could be directly compared to the relative navigation weighting $(w)$ in our model.

Predictions from our model about how animals should (in theory) balance persistence and external navigation cues (taxis) to give the highest navigational efficiency should be tested and compared to observations from empirical data. One of the most interesting model predictions is shown in Figure 2a, where in the parameter region above the $w=0.5$ contour line (corresponding to values of approximately $c_{\delta}>0.8$ for low navigation error, $c_{\phi}<0.5$, and $c_{\delta}>0.9$ for medium levels of navigation error, $0.5<c_{\phi}<0.9$ ), it is more efficient to give a higher weighting to persistence than direct navigation cues. Directly comparing empirically reported 
values of $c_{\delta}$ across the literature is known to be difficult since processing and sampling of the data can change the estimated forward persistence of an observed path (Bovet \& Benhamou, 1988; Codling \& Hill, 2005). Nevertheless, high values for $c_{\delta}$ have been reported for a wide range of species including insects and nematodes $\left(0.62 \leq c_{\delta} \leq 0.94\right.$ in Byers, 2001), elk $\left(c_{\delta}=0.68\right.$ in Fortin et al. 2005), dolphins $\left(c_{\delta}=0.8\right.$ in Bailey $\&$ Thompson, 2006), foraging seabirds $\left(c_{\delta}=\right.$ 0.885 for movement mode 3 in Boyd et al. 2014), and reindeer $\left(c_{\delta}=0.84\right.$ for exploratory movement state 2 in Langrock et al. 2014). Hence, it seems clear that many animal species are capable of moving in a highly persistent manner if they choose to do so. The relative weighting between navigation and persistence in the movement behaviour of a prairie butterfly was considered by Schultz and Crone (2001). They found that when returning to within $10-22 \mathrm{~m}$ of their home habitat they were observed to use a BCRW movement that balanced persistence with navigation with a weighting of $w=0.38$ (female) and $w=0.29$ (male). However, the authors did not explore the levels of navigation and persistence error within the observed butterfly movement paths. Fortin et al. (2005) considered the balance between forward persistence and directed movements up-slope in the winter foraging of elk. Using a log-likelihood test, they showed that a BCRW model fitted the data better than the alternative of a pure BRW or a pure CRW, and that the weighting on directional bias was $w=0.17$, implying that almost five times more weighting was given to persistence than localised up-slope directed movements. The results of Schultz and Crone (2001) and Fortin et al. (2005) provide empirical evidence that some animals do give a high weighting to persistence rather than directed movement, although the contexts are slightly different to our abstracted navigation problem. In contrast, McClintock et al. (2012) used a multi-state generalised BCRW framework to analyse and describe the movements of grey seals near to localised centres of attraction (foraging areas or haul-out sites), and found 
that when close to the centres of attraction, movement was almost entirely directed $(w>0.99)$. However, the ecological context of their study (short-term foraging within a familiar territory) is different to our problem (large-scale navigation via an external directional cue). In addition, McClintock et al. (2012) didn't directly consider that localised forward persistence and directional bias are often misclassified, especially in a short movement path, a fundamental problem highlighted by Benhamou (2006).

A number of studies have considered strategies for maximising movement efficiency in the context of foraging, although these are usually based on minimising energy expenditure rather than optimising navigation efficiency. Fortin et al. (2003) demonstrated how theories from optimal foraging related to energy maximisation did not seem to hold across varying temporal scales, with other factors influencing movement behaviour becoming more important at larger time-scales. Bartoń et al. (2009) used simulations to explore the survival of animals exploring a patchy landscape using a BCRW movement model that balanced persistence with biased movement towards patches of suitable habitat. They found that “...when an organism's ability to detect patches decreases with distance from the patch, dispersal mortality is high if the organism engages in a walk with a low degree of correlation. Thus, even if long distance detection of a patch is poor, an individual can still have a good chance of surviving dispersal if it moves using a more economical, highly correlated walk." Hence, although the model of Bartoń et al. (2009) was based on short-scale localised navigation when foraging, their conclusions seem to be consistent with the findings from our model, which is based on large-scale navigation. Further work is now needed to test our model predictions and to determine in more detail how real animals may balance persistence and taxis (and other possible mechanisms) when navigating efficiently over a range of spatio-temporal scales. 
Bailey, H. and Thompson, P. 2006. Quantitative analysis of bottlenose dolphin movement patterns and their relationship with foraging. Journal of Animal Ecology, 75: 456-465.

Bartoń K. A., Phillips, B. L., Morales, J. M. and Travis, J. M. J. 2009. The evolution of an 'intelligent' dispersal strategy: biased, correlated random walks in patchy landscapes. Oikos, 118: 309-319.

Benhamou, S. 2006. Detecting an orientation component in animal paths when the preferred direction is individual dependent. Ecology, 87: 518-528

Benhamou, S., and P. Bovet. 1992. Distinguishing between elementary orientation mechanisms by means of path analysis. Animal Behaviour, 43: 371-377.

Bode, N.W.F., J.J. Faria, D.W. Franks, J. Krause, and A.J. Wood. 2010. How perceived threat increases synchronization in collectively moving animal groups. Proceedings of the Royal Society B, 277: 3065-3070.

Bovet, P., and S. Benhamou. 1988. Spatial analysis of animals' movements using a correlated random walk model. Journal of Theoretical Biology, 131: 419-433.

Boyd, C., Punt, A.E., Weimerskirch, A., Bertrand, S. 2014. Movement models provide insights into variation in the foraging effort of central place foragers. Ecological Modelling, 286: $13-25$.

Byers, J.A. 2001. Correlated random walk equations of animal dispersal resolved by simulation. Ecology, 82: 1680-1690.

Cheung, A., S. W. Zhang, C. Stricker and M. V. Srinivasan. 2007. Animal navigation: the difficulty of moving in a straight line. Biological Cybernetics, 97: 47-61 
Codling, E.A., and N.A. Hill. 2005. Sampling rate effects on measurements of correlated and biased random walks. Journal of Theoretical Biology, 233: 573-588.

Codling, E.A., M.J. Plank, and S. Benhamou. 2008. Random walk models in biology. Journal of the Royal Society Interface, 5: 813-834.

Codling, E.A., R.N. Bearon, and G.J. Thorn. 2010. Diffusion about the mean drift location in a biased random walk. Ecology, 91: 3106-3113.

Codling, E.A., and N.W.F. Bode. 2014. Copycat dynamics in leaderless animal group navigation. Movement Ecology, 2: 11.

Codling, E.A., and N.W.F. Bode. 2016. Balancing direct and indirect sources of navigational information in a leaderless model of collective animal movement. Journal of Theoretical Biology, 394: 32-42.

Fortin, D., Fryxell, J.M., O'Brodovich, L. and Frandsen, D. 2003. Foraging ecology of bison at the landscape and plant community levels: the applicability of energy maximization principles. Oecologia, 134: 219-227.

Fortin, D., Morales, J.M. \& Boyce, M.S. 2005. Elk winter foraging at fine scale in Yellowstone National Park. Oecologia, 145: 335-343.

Gardiner, J.M., Whitney, N.M., Hueter, R.E. 2015. Smells Like Home: The Role of Olfactory Cues in the Homing Behavior of Blacktip Sharks, Carcharhinus limbatus. Integrative and Comparative Biology, 55: 495-506.

Hill, N.A., and D.-P. Häder. 1997. A biased random walk model for the trajectories of swimming micro-organisms. Journal of Theoretical Biology, 186: 503-526.

Jonsen, I.D., J. M. Flemming, and R. A. Myers. 2005. Robust state-space modelling of animal movement data. Ecology, 86: 2874-2880. 
Kareiva, P., and N Shigesada. 1983. Analyzing insect movement as a correlated random walk. Oecologia, 56: 234-238.

Langrock, R., King, R., Matthiopoulos, J., Thomas, L., Fortin, D. and Morales, J. M. 2012. Flexible and practical modeling of animal telemetry data: hidden Markov models and extensions. Ecology, 93: 2336-2342.

Langrock, R., Hopcraft, J.G.C., Blackwell, P.G., Goodall, V., King, R., Niu, M., Patterson, T.A., Pedersen, M.W., Skarin, A., Schick, R.S. 2014. Modelling group dynamic animal movement. Methods in Ecology and Evolution, 5: 190-199.

Mardia, K.V., and P.E. Jupp. 1999. Directional Statistics, John Wiley, New York, NY.

Marsh, L.M., and R. E. Jones. 1988. The form and consequences of random walk movement models. Journal of Theoretical Biology, 133: 113-131.

McClintock, B. T., King, R., Thomas, L., Matthiopoulos, J., McConnell, B. J. and Morales, J. M. 2012. A general discrete-time modeling framework for animal movement using multistate random walks. Ecological Monographs, 82: 335-349.

Nathan, R. 2008. An emerging movement ecology paradigm. Proceedings of the National Academy of Sciences, 105: 19050-19051.

Peleg, O. and L. Mahadevan. 2016. Optimal switching between geocentric and egocentric strategies in navigation. Royal Society Open Science, 3: 7.

Schultz, C.B., and E.E. Crone. 2001. Edge-mediated dispersal behaviour in a prairie butterfly. Ecology, 82: 1879-1892.

Wu, H.-i., B.-L. Li, T. A. Springer, and W. H. Neill. 2000. Modelling animal movement as a persistent random walk in two dimensions: expected magnitude of net displacement. Ecological Modelling, 132: 115-124. 


\section{Figure legends}

385

386

387

Figure 1. Navigational efficiency of the vector-weighted BCRW model, $c_{\theta}$, against weighting factor on navigation, $w$, for a range of navigation error levels, $c_{\phi}=0.1$ (black), $c_{\phi}=0.3$ (cyan), $c_{\phi}=0.6$ (gold) \& $c_{\phi}=0.9$ (blue). In all plots the solid lines represent the theoretical model predictions and the dashed lines represent the average results from simulations of 1000 individual random walkers; $w$ ranges from 0 to 1 at 0.01 intervals. Plots (a-c) show results with zero persistence error $\left(c_{\delta}=1\right)$ for walks with different total number of steps: a) 100, b) 1000 , c) 10,000. Plots (d-i) show results for 1000 steps with persistence errors d) $c_{\delta}=0.99$, e) $c_{\delta}=0.95$, f) $\left.\left.\left.c_{\delta}=0.9, \mathrm{~g}\right) c_{\delta}=0.7, \mathrm{~h}\right) c_{\delta}=0.5, \mathrm{i}\right) c_{\delta}=0.1$. Navigation and persistence errors were drawn from zero-centred wrapped Normal distributions with parameters $c_{\delta}$ and $c_{\phi}$ respectively.

Figure 2. Heat maps showing (a) the weighting factor on navigation, $w$, that leads to the predicted maximum navigation efficiency, and (b) the corresponding maximum navigation efficiency values. Plots (c) and (d) show the respective average results from simulations of 1000 individual random walkers moving for 1000 steps. In each plot, $c_{\delta}$ and $c_{\phi}$ range from 0 to 1 at 0.01 intervals. Values of $w$ ranging from 0 to 1 at 0.01 intervals were used to find the maximum navigation efficiency for each combination of $c_{\delta}$ and $c_{\phi}$. Navigation and persistence errors were drawn from zero-centred wrapped Normal distributions with parameters $c_{\delta}$ and $c_{\phi}$ respectively. 

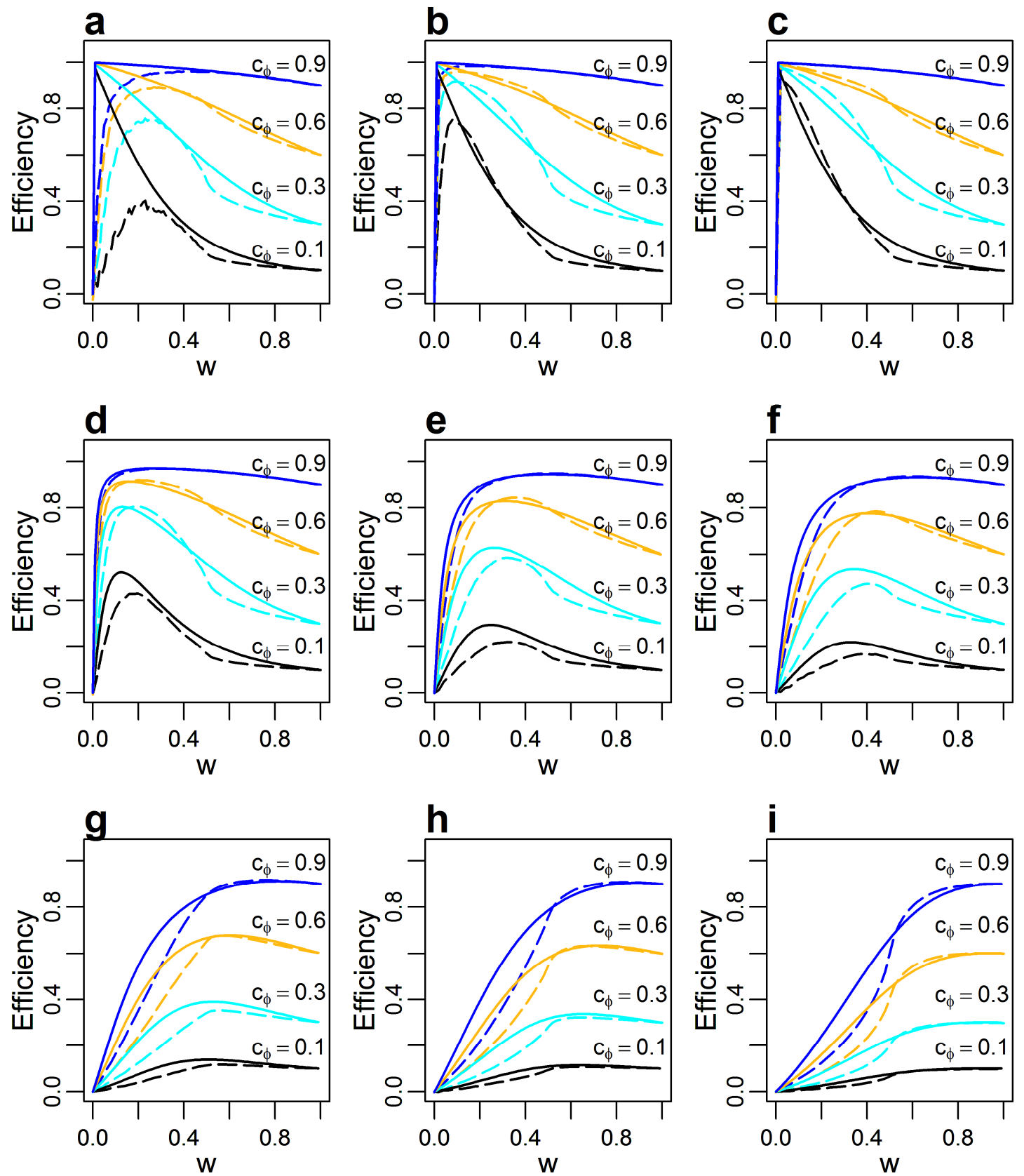

403 

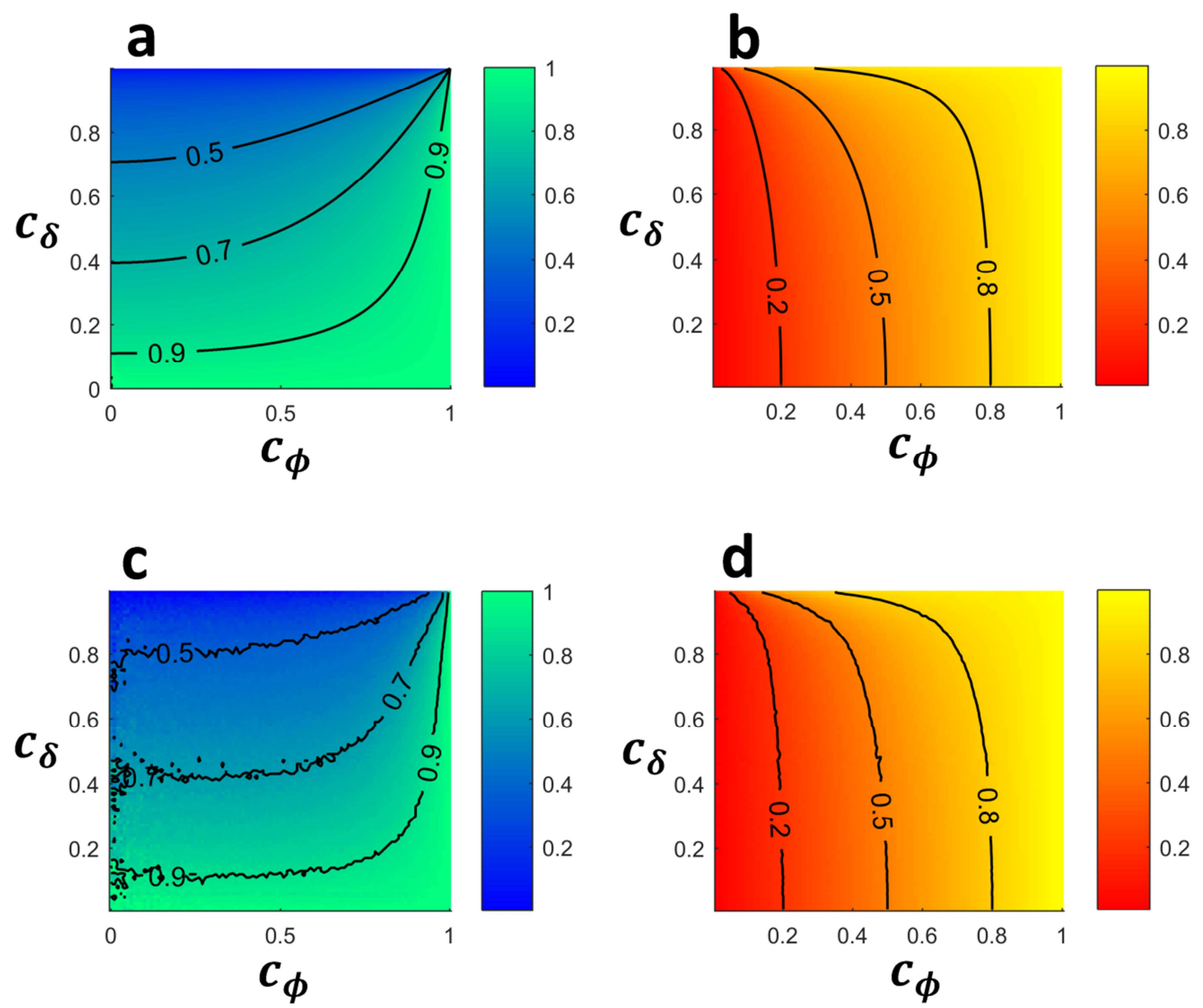

405 